\title{
Practical Plastic and Reconstructive Surgery
}

\section{Naven Olivari}

\section{Edition 2009}

Kaden Publishers, Heidelberg, Germany

No. of pages: 943

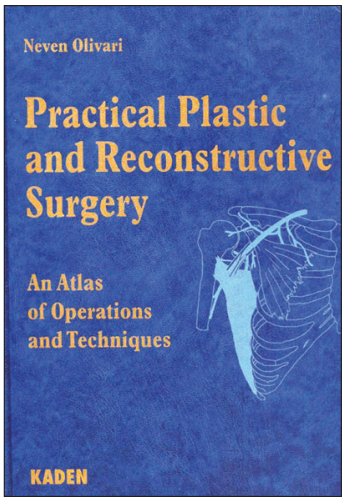

for Cleft Lip, reconstruction of lip defects, local flaps on the face and reconstruction of nose are discussed in vivid details in this section.

Section II is on 'General Principles' in which the author offers a classification of flaps and illustrates commonly used random pattern, axial pattern, myocutaneous, fasciocutaneous and free flaps. There are also lucid descriptions of Tissue expansion, Wolfe grafts and composite grafts in this section. Practical tips to correctly fix the Wolfe graft on the nasal tip and dorsum, or use a composite auricular cartilage for alar defects are remarkable; and words of caution like "Post operative cooling of the composite graft for 24 hours" and "a blue graft is a good sign and a white graft is a suspect" are instantly eye catching. Bone grafting and management of soft tissue trauma also are wonderfully illustrated in this section

Section III is on 'Head and Neck', and this is my favorite part of this book. Suggesting with absolute clarity how to cover defects in the nasal area, eyelid region, lips, rest of the cervico-facial region and the hair bearing scalp, this is the section where the author describes the surgical treatment of endocrine ophthalmopathy, an area, the author has long been renowned for. In this section, the author introduces to his readers unique instruments like the Olivari Rhinostome, which he uses for lateral osteotomy in his rhinoplasties, and which he claims, is less traumatic than the saw and more precise than the chisel.

Every deformed nose is depicted by a line diagram of the morbid anatomy of its skeleton before surgery and what was done during surgery and finally the anatomy 
of the corrected skeletal framework. Pre, per and postoperative photographs accompany these diagrams to make is very interesting to read. Words of caution for the reader are in abundance - cautioning against people with very unreasonable expectations from face lift, the author says "the face may be lifted, but the human being as a whole is not rejuvenated". He is also a bitter critic of suboptimal surgery and does not spare surgeons "who choose a 5-cm pre-auricular incision and who have the nerve to publicize this at congresses and on television as mini lift".

This section also has 'Cleft Lip, Jaw and Palate' and the author shows a clear preference to Tennyson repair with a small extra triangle a-la-Skoog, at the nostril floor. His coverage of alveolus with buccal flap instead of gingival tissue is however not destined to allow eruption of teeth through it. Facial nerve pareses and repair of ear and conchal defects also find a place in this section.

Section IV depicts 'Chest and Chest Wall' and in this the author describes post mastectomy breast reconstruction in great details. Use of implants, pedicled Latissimus dorsi, pedicled TRAM, free TRAM, turbo TRAM and free gluteus maximus - their indications, contra-indications, planning, surgical technique and complications are described in vivid details. Correction of inverted nipple by author's own technique, treatment of hydradenitis suppurativa and use of free tissue transfer for massive chest wall and axillary defects also find a mention in this section.

Section V consists of 'Abdomen, gluteal region and genitelia' and in this the author has tackled the problem of large abdominal hernias and highlighted the use of Prolene mesh and Ramirez procedure. The treatment of obesity - liposuction, abdominoplasty and body lift are also mentioned in this section as are the treatment of pressure sores, pilonidal sinus, penile contractures, perineal defects and epispadias.

Section VI is based on 'Skin and soft tissue defects in the extremities' and in this the author talks about various types of flaps used in limb reconstruction. He also discusses 'amniotic band syndrome' in great details, dividing it into grades I to $\mathrm{V}$ defining surgery for each grade. The highlight of this section however is his 'Principles of replantation surgery', in which, he lucidly elucidates the pre-operative treatment of the amputated part, indications of replantation, types of amputation, pre operative planning, surgery, post operative monitoring and possible complications.

Section VII is on 'Surgical therapy of burn injuries' which not only comprises detailed synopsis of the pathophysiology of burn, but also portrays a classification of burn trauma and detailed treatment options of both generalized body surface and specialized areas like the hand.

The fact that Prof. Naven Olivari has not only authored the book, but has also drawn each and every illustration obviates any possible communication gap between the author and his artist/illustrator. Published originally in German, the book is now available in English for the benefit of a larger audience, which it surely deserves. The 943 page, hard bound single volume book has been published by Kaden Publishers of Heidelberg, Germany. This is a book which is a must for every Plastic Surgery library for the author's passion and brilliance and the book's simplicity and clarity.

\section{Surajit Bhattacharya}

Editor, Indian Journal of Plastic Surgery surajitb@sancharnet.in 